
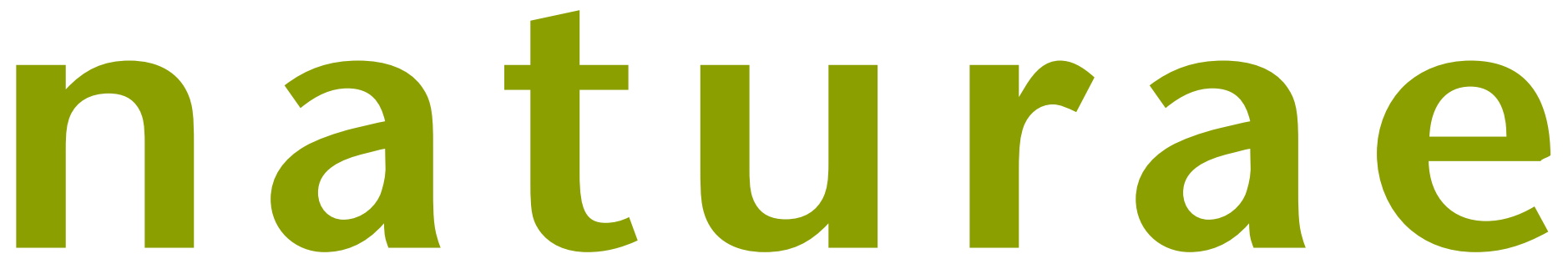

$2021 \cdot 19$

REVER 10 - 10ĖME COLLOQUE DU RÉSEAU D'ÉCHANGE

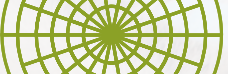
ET DE VALORISATION EN ÉCOLOGIE DE LA RESTAURATION PARIS, 19-21 MARS 2019

Édité par Philippe GOURDAIN

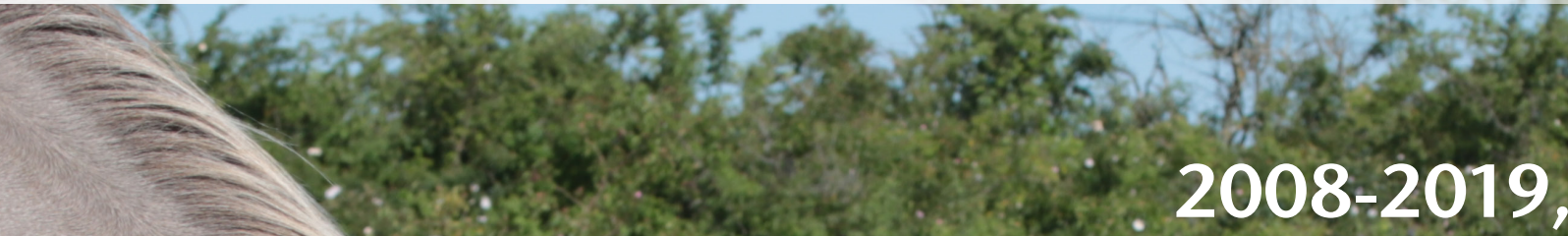
plus d'une décennie d'échanges et de débats autour de la restauration écologique en Frañce

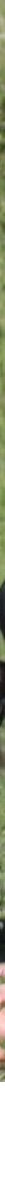

Whan

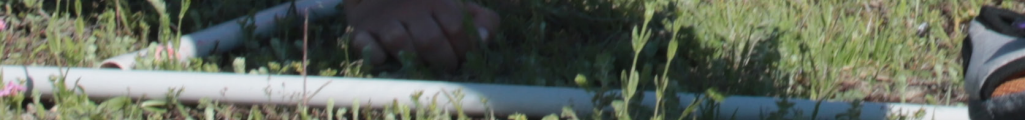
7. - Mart. 2021 (19) a publie le \& septembre 2021 . $x^{2}$ An ww.revue-naturae.fr

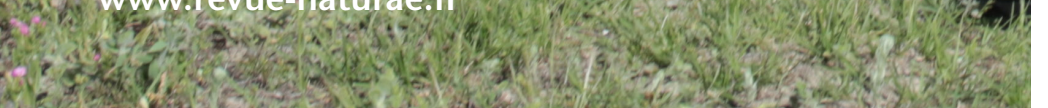

PUBLICATIONS SCIENTIFIOUES
MUSËUMW (8) NATURELE W)

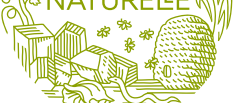


Directeur de LA Publication / Publication diRECTOR: Bruno David,

Président du Muséum national d'Histoire naturelle

RÉDACTEUR EN CHEF / EDITOR-IN-CHIEF: Jean-Philippe Siblet

ASSISTANTE DE RÉDACTION / ASSISTANT EDITOR: Sarah Figuet (naturae@mnhn.fr)

Mise EN PAGE / PAGE LAYOUT: Sarah Figuet

COMITÉ SCIENTIFIQUE / SCIENTIFIC BOARD:

Luc Abbadie (UPMC, Paris)

Luc Barbier (Parc naturel régional des caps et marais d'Opale, Colembert)

Aurélien Besnard (CEFE, Montpellier)

Vincent Boullet (Expert indépendant flore/végétation, Frugières-le-Pin)

Hervé Brustel (École d'ingénieurs de Purpan, Toulouse)

Patrick De Wever (MNHN, Paris)

Thierry Dutoit (UMR CNRS IMBE, Avignon)

Éric Feunteun (MNHN, Dinard)

Romain Garrouste (MNHN, Paris)

Grégoire Gautier (DRAAF Occitanie, Toulouse)

Olivier Gilg (Réserves naturelles de France, Dijon)

Frédéric Gosselin (Irstea, Nogent-sur-Vernisson)

Patrick Haffner (UMS PatriNat, Paris)

Frédéric Hendoux (MNHN, Paris)

Xavier Houard (OPIE, Guyancourt)

Isabelle Leviol (MNHN, Concarneau)

Francis Meunier (Conservatoire d'espaces naturels - Hauts-de-France, Amiens)

Serge Muller (MNHN, Paris)

Francis Olivereau (DREAL Centre, Orléans)

Laurent Poncet (UMS PatriNat, Paris)

Nicolas Poulet (OFB, Vincennes)

Jean-Philippe Siblet (UMS PatriNat, Paris)

Laurent Tillon (ONF, Paris)

Julien Touroult (UMS PatriNat, Paris)

COUVERTURE / COVER:

Suivi des effets de la ré-introduction de chevaux Konik Polski (Equus ferus caballus Boddaert, 1785) sur la végétation des digues du canal de Donzère-Mondragon (Vaucluse, France). Crédit photo: IMBE.
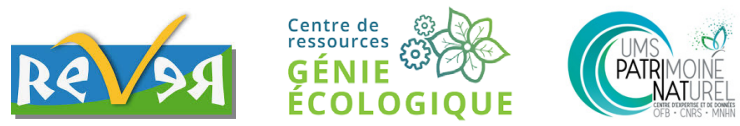

Naturae est une revue en flux continu publiée par les Publications scientifiques du Muséum, Paris Naturae is a fast track journal published by the Museum Science Press, Paris

Les Publications scientifiques du Muséum publient aussi / The Museum Science Press also publish:

Adansonia, Zoosystema, Anthropozoologica, European Journal of Taxonomy, Geodiversitas, Cryptogamie sous-sections Algologie, Bryologie, Mycologie, Comptes Rendus Palevol.

Diffusion - Publications scientifiques Muséum national d'Histoire naturelle

CP 41 - 57 rue Cuvier F-75231 Paris cedex 05 (France)

Tél.: 33 (0)140794805 / Fax: 33 (0)1 40793840

diff.pub@mnhn.fr / https://sciencepress.mnhn.fr

(c) Publications scientifiques du Muséum national d'Histoire naturelle, Paris, 2021

ISSN (électronique / electronic): 1638-9387 


\title{
2008-2019, plus d'une décennie d'échanges et de débats autour de la restauration écologique en France
}

\author{
Thierry DUTOIT \\ Institut méditerranéen de Biodiversité et d'Écologie marine et continentale (IMBE), \\ Avignon Université, CNRS, IRD, Aix-Marseille Université, \\ IUT Avignon, site Agroparc, boîte postale 61207, F-84911 Avignon cedex 09 (France) \\ thierry.dutoit@univ-avignon.fr \\ Sébastien GALLET \\ Laboratoire Géoarchitecture, Territoires, Urbanisation, Biodiversité, Environnement, \\ Université de Bretagne Occidentale CS 93837, F-29238 Brest cedex 3 (France) \\ sebastien.gallet@univ-brest.fr
}

Alma HECKENROTH Laboratoire Population Environnement Développement, Aix-Marseille Université, case 103, place Victor Hugo, CS80249, F-13331 Marseille cedex 03 (France) alma.heckenroth@univ-amu.fr

Élise BUISSON Institut méditerranéen de Biodiversité et d'Écologie marine et continentale (IMBE), Avignon Université, CNRS, IRD, Aix-Marseille Université, IUT Avignon, site Agroparc, boîte postale 61207, F-84911 Avignon cedex 09 (France) elise.buisson@univ-avignon.fr

Soumis le 20 mai 2020 | Accepté le 2 novembre 2020 | Publié le 8 septembre 2021

MOTS CLÉS Histoire, concepts, Réseau d'Échanges et de Valorisation en Ecologie de la Restauration (REVER) retour d'expérience.
Dutoit T., Gallet S. Heckenroth A. \& Buisson É. 2021 - 2008-2019, plus d'une décennie d'échanges et de débats autour de la restauration écologique en France, in Gourdain P. (éd.). REVER 10 - 10 ème Colloque du Réseau d'Échange et de Valorisation en Écologie de la Restauration, Paris, 19-21 mars 2019. Naturae 2021 (19): 271-276. https://doi.org/10.5852/ naturae2021a19

\section{RÉSUMÉ}

Le colloque REVER 10 «Restaurer ou reconquérir» organisé en mars 2019 par le MNHN et l'association REVER, a été l'occasion de faire un point d'étape sur le développement de l'écologie de la restauration et de la restauration écologique, au moment où débute la «décennie de la restauration des écosystèmes» décrétée par l'ONU. En France, le développement de la restauration écologique a été accompagnée par le réseau REVER créé en 2008 sur le modèle de la SER (Society for Ecological Restoration) qui favorise, notamment par l'organisation de colloques annuels, les échanges entre acteurs techniques et scientifiques ainsi que la circulation des connaissances, des concepts et des pratiques. Ces échanges ont été particulièrement importants au moment où, sur la même période, la recherche et les retours d'expériences ont conduit à une évolution et à un confortement des concepts par de nombreux débats scientifiques autour notamment des notions d'écosystèmes de référence, de nouveaux écosystèmes et de restauration passive. Aujourd'hui plus que jamais, même si la préservation doit rester une priorité, les enjeux de la restauration rendent indispensable le renforcement tant de l'acquisition de connaissances que de l'action. Ainsi la diffusion scientifique, la construction d'outils d'évaluation et d'aide à la décision et les échanges autour de retours d'expériences restent primordiaux et le réseau REVER, en lien avec les structures internationales, a un rôle indéniable à jouer. 


\begin{abstract}
2008-2019, more than a decade of exchanges and debates around ecological restoration in France. The REVER 10 conference "Restoring or recovering" organized by MNHN (National Museum of Natural History) and REVER association in March 2019, was an opportunity to review the development of restoration ecology and ecological restoration, as the "Decade of Ecosystem Restoration" was being proclaimed by the UN. In France, the development of ecological restoration has been guided by the REVER association, a network created in 2008, on the model of the SER (Society for Ecological Restoration). It contributes to organizing annual conferences, promotes exchanges between technical and scientific stakeholders and the diffusion of knowledge, concepts and practices. These exchanges were particularly important at a time when, over the same period, research and feedbacks from restoration projects had been leading to an evolution and a strengthening of concepts through numerous scientific debates around, in particular, the notion of reference ecosystems, novel ecosystems and passive restoration. Today more than ever, even if conservation must remain a priority, the challenges we face in ecological restoration make it essential to strengthen both the acquisition of knowledge and restoration actions. Thus, scientific dissemination, the design of evaluation tools and of decision support systems and exchanges around restoration project feedbacks remain essential in which REVER along with international structures have an undeniable role to play.
\end{abstract}

\section{INTRODUCTION}

Depuis une trentaine d'années, face au constat de la dégradation des écosystèmes, la restauration écologique apparait comme un outil indispensable au maintien de la biodiversité en complément des actions de conservation. Le développement de sa mise en œuvre s'est fait progressivement et en parallèle de la construction de concepts au sein d'une nouvelle discipline scientifique: l'écologie de la restauration.

Écologie de la restauration et restauration écologique sont indissociables et l'une ne peut, ni ne doit, se déployer sans l'autre. La théorie doit accompagner la pratique mais doit s'appuyer sur celle-ci pour alimenter ses réflexions; la pratique doit s'adapter à l'évolution des connaissances mais doit aussi interpeler la recherche sur la base de ses retours d'expérience. Ainsi les échanges entre les acteurs multiples impliqués dans la restauration écologique sont fondamentaux afin que les actions menées permettent d'atteindre un objectif commun, préserver la biodiversité et reconquérir des espaces où elle peut s'exprimer en favorisant la résilience des écosystèmes dans toute leur diversité.

C'est dans ce contexte que se sont créés des réseaux visant à favoriser les échanges entre chercheurs et praticiens, tout d'abord à l'échelle internationale avec la création de SER (Society for Ecological Restoration) au milieu des années 80, déclinée ensuite à l'échelle européenne au début des années 2000 et enfin à l'échelle nationale avec la création, en 2008, de REVER (Réseau d'Échanges et de Valorisation en Écologie de la Restauration).

Le colloque REVER 10 «Restaurer ou Reconquérir», qui s'est tenu en Mars 2019 (Fig. 1) et dont sont issus les articles de cette thématique, est l'occasion de revenir sur la dernière décennie, qui a vu le développement des opérations de restauration écologique, et qui a été aussi particulièrement riche d'échanges et de débats scientifiques.

\section{UNE DÉCENNIE D’ÉCHANGES}

Après une première édition en 2009, suivie en 2010 d'un colloque de SER-Europe à Avignon, les éditions des journéesateliers du réseau REVER (né en 2008) se sont succédées annuellement et ont permis un véritable tour de France de la restauration écologique passant par Avignon, Brest, Metz, Lyon, Rouen, Strasbourg, Bordeaux, Arras et Arles. Au cours de chacune de ces éditions, les échanges formels et informels ont été riches et ont permis d'aborder des sujets très variés comme cela a déjà été présenté dans cette revue (Gallet et al. 2017). Elles ont également permis de mettre en relation les acteurs de la restauration écologique dans un subtil dosage entre approches locales et nationales, pratiques et théoriques.

Pour achever cette première décennie et marquer cet anniversaire, le 10e colloque du réseau REVER intitulé « REVER ou Reconquérir?» s'est tenu à Paris du 19 au 21 mars 2019 en partenariat avec le Muséum national d'Histoire naturelle (MNHN - UMS PatriNat) et le Centre de ressources du génie écologique de l'Office français pour la Biodiversité (OFB). Exceptionnellement, les communications se sont déroulées sur deux journées complètes. Organisées autour de quatre grands thèmes (milieux ouverts, végétalisation et translocation, cours d'eau et zones humides et outils socio-économiques), les 23 communications orales et les 17 posters ont permis de balayer un vaste éventail de problématiques, approches et situations, de succès mais aussi, car elles sont tout aussi révélatrices, de situations d'échecs ou de mises en difficulté. Deux tables rondes ont en outre permis d'offrir un temps d'échanges sur les sujets des espèces exotiques envahissantes et de la formation dans le domaine de la restauration écologique. Ce dernier sujet a permis notamment de confronter les attentes des professionnels avec l'offre de formation, notamment universitaire. Elle a montré une nouvelle fois l'importance des échanges entre formateurs et recruteurs afin que les premiers 

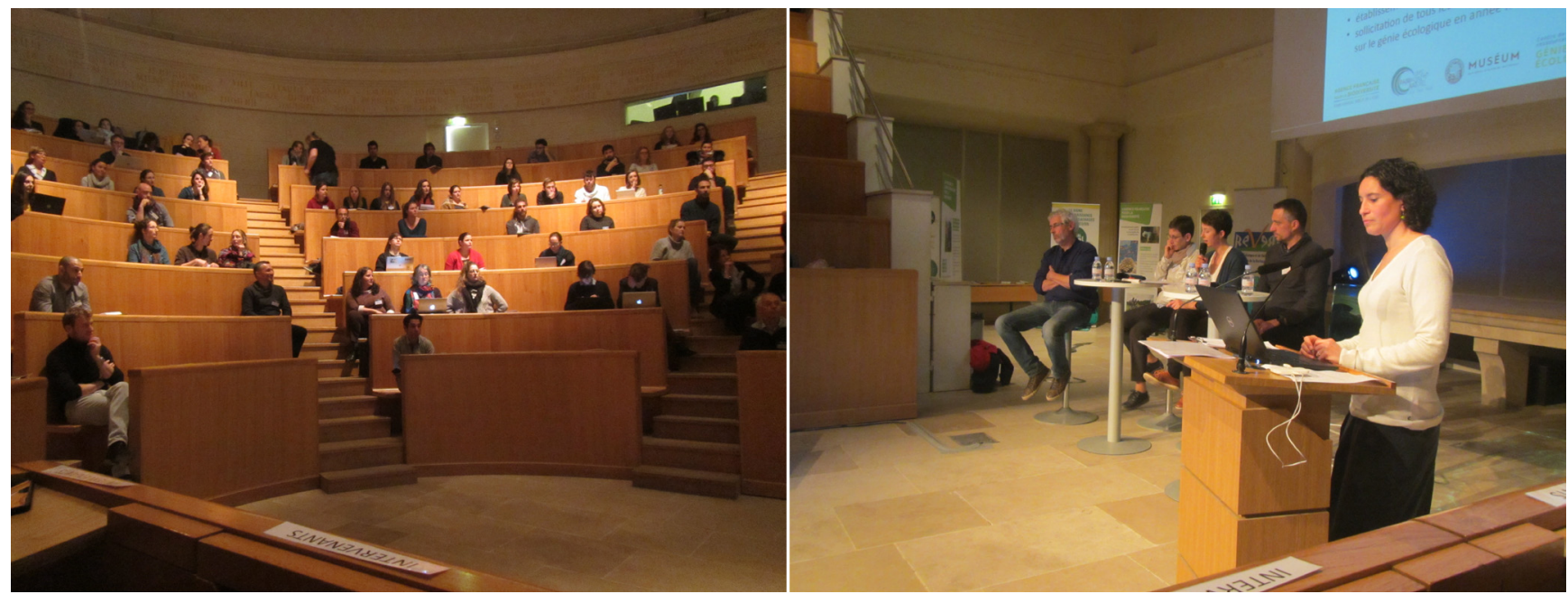

FIG. 1. - Participants et quelques intervenants lors du colloque REVER 10 dans l'amphithéâtre du Muséum national d'Histoire naturelle. Crédits photos: P. Gourdain.

assurent au mieux l'employabilité de leurs diplômés, mais aussi que les seconds prennent conscience de la richesse des formations existantes et des contraintes et limites à ce que les organismes de formation peuvent offrir.

Cette dixième édition, par le nombre de participants (214, un record d'affluence pour REVER), par le cadre exceptionnel dans lequel elle s'est déroulée grâce aux partenariats avec le MNHN et l'OFB, mais aussi par la qualité et la richesse des présentations et des débats, restera certainement une édition de référence.

\section{UNE DÉCENNIE DE DÉBATS SCIENTIFIQUES}

Comme toute discipline scientifique, l'écologie de la restauration est, depuis sa création, traversée par divers courants de pensées issus de résultats expérimentaux originaux, de propositions de nouvelles théories ou encore de l'actualité qui met brutalement en exergue des recherches ou des résultats demeurés jusqu'à présent plus ou moins confidentiels au sein de la communauté des chercheurs et ingénieurs spécialistes du domaine concerné.

REVER 10 a été l'occasion de revenir sur l'évolution des concepts et théories en écologie de la restauration durant la décennie qui a suivi la création du réseau en 2008. Le moins que l'on puisse dire, c'est que cette période fut riche en évènements ayant conduit à de vifs débats et une profonde remise en cause de l'orthodoxie interventionniste de la restauration d'écosystèmes historiques au profit de la restauration passive, au nom de la libre expression de la naturalité des nouveaux écosystèmes ou encore plus récemment, de leur ré-ensauvagement.

Il est vrai que les décennies précédentes avaient surtout été consacrées, et cela est relativement classique pour une discipline scientifique émergente, à la définition des concepts et du vocabulaire de base de la restauration écologique. Il fallait ainsi être capable de distinguer une restauration sensu stricto, d'une réhabilitation ou encore d'une réaffectation (Bradshaw 1987). Les débats furent notamment intenses autour de ces notions dans la décennie des années 80 , avant qu’ils ne soient supplantés dans les années 90 par ceux tournant autour de la nécessité ou pas de définir un écosystème de référence en matière de restauration écologique (Aronson et al. 1995). Tout système biologique est cependant en perpétuelle évolution et il est difficile d'en prédire la direction, notamment dans un monde qui subit des changements globaux climatiques ou d'usages. La restauration sensu stricto de toutes les composantes et fonctions d'écosystèmes historiques de référence apparaît alors d'autant plus difficile à atteindre. Si ces idées étaient déjà d'actualité à la fin du siècle précédent, elles prennent particulièrement corps à la fin des années 2000 suite à la parution d'articles provenant de méta-analyses issues de centaines de retours d'expériences d'opérations de restauration menées à travers le monde. Cellesci ont certes démontré les aspects bénéfiques des interventions de restauration écologique, mais aussi leur incapacité à restaurer l'intégralité des composantes et fonctions détruites ou dégradées quel que soit l'écosystème considéré (Rey-Benayas et al. 2009; Suding 2011; Moreno-Mateos et al. 2012).

C'est notamment de ces résultats qu'apparaîtra au cours des dix dernières années une révision de l'état dit dégradé ou altéré des écosystèmes (Hobbs 2016). Celle-ci fait notamment suite à l'émergence du concept de "nouvel écosystème» (Hobbs et al. 2009 ; 2013) défini quant à lui comme un habitat et une biocénose d'un socio-écosystème qui, en raison des activités anthropiques, diffèrent de ceux qui existaient historiquement. Suite à l'abandon des interventions humaines, ceux-ci seront toutefois capables de s'auto-organiser et de présenter des propriétés émergentes mais difficilement prédictibles du fait de l'inexistence d'écosystèmes analogues et de l'impact attendu des changements globaux. La restauration de l'intégralité de ces écosystèmes serait alors inatteignable faute de connaissances scientifiques, de moyens techniques mais aussi des coûts socioéconomiques de ces opérations (Jackson \& Hobbs 2009). Remettant en cause la restauration active basée sur le pilotage d'une trajectoire de référence vers un écosystème historique, ce nouveau concept a entrainé de vifs échanges dans la littérature scientifique au tournant des décennies 2000-2010 (Aronson et al. 2014; Hobbs et al. 2014; Murcia et al. 2014). 
La virulence du débat concernait notamment l'intégration des facteurs socio-économiques comme seuil d'irréversibilité pour la réussite d'une opération de restauration, mais aussi le risque que ce concept donne carte blanche aux aménageurs pour la destruction de tout écosystème sans restauration compensatoire, puisque les nouveaux écosystèmes produits auraient alors de nouvelles potentialités même si celles-ci sont encore aujourd'hui largement méconnues...

Si l'apparition de ce concept a produit un certain désarroi dans la communauté des chercheurs en écologie de la restauration (Hobbs 2018), de nouvelles méta-analyses ont confirmé que la restauration active n'entraîne pas forcément une récupération plus rapide ou plus complète que la libre expression après la fin des causes de dégradation (Jones et al. 2018). Les résultats de ces travaux sont cependant à interpréter avec prudence car peu d'études ont comparé différentes actions de restauration au même endroit et sur des durées significatives. En effet, les variations du succès de restauration sont aussi fortement dépendantes des conditions environnementales dans lesquelles elles ont été mises en place (Stuble et al. 2017). Enfin, la restauration passive ne fait pas systématiquement appel à un écosystème de référence qui permettrait de mesurer le chemin qui reste encore à parcourir pour un succès total (Marchand et al. 2021). C'est aussi le cas du ré-ensauvagement dont les objectifs sont de récupérer de la «nature sauvage» dans des paysages écologiquement dégradés. À la différence de la restauration écologique sensu stricto, les fonctions écosystémiques présentes et futures seraient dans ce cas plus importantes que les fonctions historiques de référence (Perino et al. 2019; du Toit \& Petorelli 2019). Faute de restauration à l'identique de l'écosystème historique, le nouvel écosystème est ici accepté pour son potentiel qui peut alors être augmenté par l'introduction d'espèces accélérant le ré-ensauvagement (grands herbivores et leurs prédateurs notamment) au détriment cependant de l'intégration de ce type d'opérations dans le contexte socio-économique actuel, ne serait-ce que par les surfaces nécessaires à la réussite de tels projets (plusieurs milliers à dizaines de milliers d'hectares).

En conclusion, les vifs courants qui ont traversé et qui traversent encore l'écologie de la restauration sont à l'image des enjeux actuels autour de la conservation d'un environnement planétaire viable, exacerbés par les changements climatiques ou la toute récente pandémie de Covid-19. Contrairement aux physiciens et cosmologistes, les écologues ne sont cependant pas à la recherche d'une théorie unifiée car ils savent de par leur base naturaliste que seule la multiplicité de nos rapports avec la nature, y compris dans le domaine de la restauration, sera à l'origine de la diversité des systèmes biologiques et donc du maintien de la résilience globale de la nature.

\section{UNE NOUVELLE DÉCENNIE CRUCIALE}

Si 2019 marque les dix ans du réseau REVER, elle marque aussi le début d'une nouvelle décennie, désignée le 1er mars 2019 comme «la décennie de la restauration des écosystèmes " par l'ONU qui reconnaît ainsi le rôle crucial de la restauration écologique comme un outil d'amélioration des conditions environnementales et de mise en valeur des communautés humaines. Aujourd'hui plus que jamais, il nous faut favoriser la résilience des écosystèmes à travers des démarches actives ou passives de restauration écologique, comme une stratégie d'adaptation humaine nécessaire pour répondre aux enjeux des changements globaux. Cela doit passer par une appréhension et une caractérisation objective des perturbations et dégradations anthropiques, pas seulement centrées sur des espèces ou des habitats emblématiques, mais aussi sur l'ensemble des compartiments biotiques et abiotiques - notamment les sols, bien souvent oubliés, et pourtant supports essentiels du vivant et de services écosystémiques. Cela passe également par l'anticipation des perturbations futures, l'interdisciplinarité et la mise en place de partenariats et de collaborations. Le développement de la restauration écologique ne peut se faire qu'à partir de la diffusion des connaissances des milieux experts vers les sociétés civiles, par l'échange d'outils, les retours d'expériences, mais aussi la formation aux concepts, aux pratiques et aux métiers de la restauration écologique. Dans ce contexte présent et à venir, REVER, a définitivement un rôle à jouer et les futures éditions des colloques REVER devront plus que jamais accompagner ce développement.

Néanmoins, nous ne le rappellerons jamais assez: la limitation des impacts de nos activités, des aménagements, de l'artificialisation et plus largement de notre empreinte écologique, est un facteur crucial qui doit aller de pair avec des compromis sociétaux éthiques et pragmatiques. En effet, même si des actions sont développées pour restaurer les écosystèmes a posteriori, nous savons que nous ne parviendrons jamais aux degrés de complexité et de fonctionnalité écosystémiques qui précédaient les dégradations et que le temps nous est compté pour relever les défis présents et à venir dans un monde fini où notre empreinte écologique ne cesse globalement de se renforcer.

\section{DES RETOURS D'EXPÉRIENCES ET DES ACTIONS POUR AVANCER}

Les enjeux actuels de conservation et de restauration impliquent une réflexion globale sur nos activités et impacts sur l'environnement. Néanmoins cette réflexion ne doit pas se faire aux dépends de l'action et de la compréhension des processus de restauration.

Le colloque REVER est l'occasion d'avoir une vision globale de l'actualité nationale en restauration écologique. Les articles présentés dans ce numéro spécial, issus de communications au colloque REVER 10, illustrent la diversité de ces travaux et opérations, mais aussi des approches mises en œuvre et des façons de les aborder. Ils concernent une variété d'écosystèmes, depuis les milieux méditerranéens jusqu'aux rivières normandes en passant par les landes bretonnes et les bords du Rhin. Ils abordent l'analyse des dynamiques de résilience après des opérations de restauration actives impliquant par exemple des transferts de biomasse dans des système prairiaux 
(Garrouj et al. sous presse) ou de landes (Sawtschuk et al. sous presse) ou encore la recolonisation spontanée de berges après l'élimination d'un aménagement fluvial majeur (Ravot et al. sous presse). Ils analysent aussi les facteurs qui vont influencer ces processus dans le cas par exemple de la plaine de Crau (Vidaller et al. sous presse). L'exemple des garrigues (Heckenroth et al. sous presse) illustre, quant à lui, le lien entre deux enjeux environnementaux: la conservation de la biodiversité et la pollution des sols.

Si le partage des expériences par la publication d'articles et les échanges est fondamental il est également important de disposer d'outils qui, sur la base de celles-ci, permettent d'accompagner les acteurs de la restauration tant dans la mise en place de suivis que dans l'évaluation des gains écologiques. Ce sont ces démarches qu'illustrent notamment les deux derniers articles (Vivier et al. sous presse; Bezombes et al. sous presse).

Ces articles, comme l'ensemble des communications faites pendant le colloque REVER 10 et comme ceux des neuf précédentes éditions, doivent alimenter nos réflexions et nous permettre d'améliorer nos actions futures et ce, que nous soyons praticiens, décideurs, chercheurs ou étudiants. Ne doutons pas que les futures éditions seront à la hauteur de celle-ci.

\section{Remerciements}

Les auteurs remercient tous les organisateurs et financeurs des journées REVER 10, en particulier l'UMS PatriNat (OFBCNRS-MNHN) et l'Office français de la Biodiversité, ainsi que Philippe Gourdain pour sa relecture et ses corrections.

\section{RÉFÉRENCES}

Aronson J., Dhillion S. \& Le Floc'H E. 1995. — On the need to select an ecosystem of reference, however imperfect: A Reply to Pickett and Parker. Restoration Ecology 3: 1-3. https://doi. org/10.1111/j.1526-100X.1995.tb00069.x

Aronson J., Murcia C., Kattan G., Moreno-Mateos D., Dixon K. \& Simberloff D. 2014. - The road to confusion is paved with novel ecosystem labels: a reply to Hobbs et al. Trends in Ecology and Evolution 29 (12): 646-647. https://doi. org/10.1016/j.tree.2014.09.011

Bezombes L., Barillier A., Gouraud V. \& Spiegelberger T. (sous presse). - Améliorer les estimations de gains de biodiversité apportés par les mesures compensatoires: un retour d'expérience des actions de restauration menées sur l'île de Kembs , in Gourdain P. (éd.), REVER 10 - 10ème Colloque du Réseau d'Échange et de Valorisation en Écologie de la Restauration, Paris, 19-21 mars 2019. Naturae 2022.

BRADSHAW A. D. 1987. - Restoration: an ecological acid test, in JORDAN W. R., GILPIN M. \& ABER J. D. (éds), Restoration Ecology: A Synthetic Approach to Ecological Research. Cambridge University Press, $342 \mathrm{p}$.

Gallet S., Jaunatre R., Regnery B., Alignan J.F., Bernez I., Combroux I., Glasser T., HeCKenroth A., Jund S., Lelièvre S., Malaval S., Moussard S., Muller I., VéCrin-Stablo M.-P. \& BUISSON E. 2017. — L'écologie de la restauration en France. Dynamique actuelle et rôle d'un réseau multi-acteurs, REVER. Naturae 2017 (7): 1-11.

Garrouj M., Benot M.-L., Corcket E., Marchand L. \& ALARD D. (sous presse). - Processus initiaux impliqués dans l'installation d'une communauté végétale et représentation de la dynamique de restauration écologique d'une prairie alluviale par transfert de foin et gestions différenciées, in GOURDAIN P. (éd.), REVER 10 - 10ème Colloque du Réseau d'Échange et de Valorisation en Écologie de la Restauration, Paris, 19-21 mars 2019. Naturae 2021.

Heckenroth A., Laffont-Schowb I., Baumberger T., Prudent P., Le Mire-Pecheux L., Baumel A., Dumas E., Miché L., Tatoni T., Mirleau P. \& Affre L. (sous presse). - Vers la restauration écologique du littoral remarquable du Parc national des Calanques: comment allier stabilisation des sols contaminés et préservation d'Astragalus tragacantha L., in GOURDAIN P. (éd.), REVER 10 - 10ème Colloque du Réseau d'Échange et de Valorisation en Écologie de la Restauration, Paris, 19-21 mars 2019. Naturae 2022.

HobBs R. J. 2016. — Degraded or just different? Perceptions and value judgements in restoration decisions. Restoration Ecology 24 (2): 153-158. https://doi.org/10.1111/rec.12336

HobBs R. J. 2018. - Restoration Ecology's silver jubilee: innovation, debate and creating a future for restoration ecology. Restoration Ecology 26 (5): 801-805. https://doi.org/10.1111/rec.12863

Hobbs R. J., Higgs E. \& Harris J. A. 2009. — Novel ecosystems: implications for conservation and restoration. Trends in Ecology and Evolution 24 (11): 599-605. https://doi.org/10.1016/j. tree.2009.05.012

Hobbs R. J., Higgs E. S. \& Hall C. M. 2013. - Novel ecosystems: Intervening in the new ecological world order. Wiley Blackwell, Chichester, $384 \mathrm{p}$.

Hobbs R. J., Higgs E. \& Harris J. A. 2014. — Novel Ecosystems: Concept or Inconvenient Reality? A response to Murcia et al. Trends in Ecology and Evolution 29 (12): 645-646. https://doi. org/10.1016/j.tree.2014.09.006

JACKSON S. T. \& HoBBS R. J. 2009. - Ecological restoration in the light of ecological history. Science 325 (5940): 567-569. https:// doi.org/10.1126/science.1172977

Jones H. P., Jones P. C., Barbier E. B., Blackburn R. C., Rey Benayas J. M., Holl K. D., McCrackin M., Meli P., MonToya D. \& Moreno Mateos D. 2018. - Restoration and repair of Earth's damaged ecosystems. Proceedings of the Royal Society B: Biological Sciences 285 (1873). https://doi.org/10.1098/ rspb.2017.2577

Marchand L., Castagneyrol B., Jimenez J. J., Rey Benayas J. M., Benot M. L., Martinez-Ruiz C., Alday J., Jaunatre R., Dutoit T., Buisson E., Mench M., Alard D., Corcket E. \& Comin F. 2021. - Conceptual and methodological issues in estimating the success of ecological restoration. Ecological Indicators 123 (107362). https://doi.org/10.1016/j.ecolind.2021.107362 Moreno-Mateos D., Power M. E., Comín F. A. \& YockTeng R. 2012. - Structural and functional loss in restored wetland Ecosystems. PLOS Biology 10 (1): e1001247. https://doi.org/10.1371/ journal.pbio. 1001247

Murcia C., Aronson J., Kattan G., Moreno-Mateos D., Dixon K. \& Simberloff D. 2014. - A critique of the Novel Ecosystems concept. Trends in Ecology and Evolution 29 (10): 548-553. https://doi.org/10.1016/j.tree.2014.07.006

Perino A., Pereira H. M., Navarro L. M., Fernández N., Bullock J. M., Ceaușu S., Cortés-Avizanda A., van Klink R., Kuemmerle T., Lomba A., Pe’er G., Plieninger T., Rey Benayas J. M., SANdom C. J., Svenning J. C. \& WheEler H. E. 2019. - Rewilding complex ecosystems. Science 364 (6438): eaav5570. https://doi.org/10.1126/science.aav5570

Ravot C., Dufour S., Le Coeur D. \& Bernez I. (sous presse). Biodiversité comparée de la végétation spontanée précoce des rives de cinq affluents à celle du cours principal de la Sélune dans la vallée renaturée de Vezin suite à l'arasement du barrage (Normandie), in GourdaIn P. (éd.), REVER 10 - 10ème Colloque du Réseau d'Échange et de Valorisation en Écologie de la Restauration, Paris, 19-21 mars 2019. Naturae 2021.

Rey-Benayas J. M., Newton A. C., Diaz A. \& Bullock J. M. 
2009. - Enhancement of biodiversity and ecosystem services by ecological restoration: a meta-analysis. Science 325 (5944): 1121-1124. https://doi.org/10.1126/science.1172460

Stuble K. L., Fick S. E \& Young T. P. 2017. - Every restoration is unique: testing year effects as drivers of initial restoration trajectories. Journal of Applied Ecology 54 (4): 1051-1057. https:// doi.org/10.1111/1365-2664.12861

Sawtschuk J., Gourdain P., Delzons O., Larzilliere A. QuENOT F. \& COÏC T. (sous presse). - Quelles trajectoires de réhabilitation pour la décharge de l'île d'Ouessant (29): retour sur dix années de recherche-action, in Gourdain P. (éd.), REVER 10 - 10ème Colloque du Réseau d'Échange et de Valorisation en Écologie de la Restauration, Paris, 19-21 mars 2019. Naturae 2021.

SUDING K. 2011. - Toward an era of restoration in ecology: sucesses, failures and opportunities ahead. Annual Review of Ecology, Evolution and Systematics 42: 465-487. https://doi.org/10.1146/ annurev-ecolsys-102710-145115
Toit J. T. Du \& PetTorelli N. 2019. — The differences between rewilding and restoring an ecologically degraded landscape. Journal of Applied Ecology 56: 2467-2471. https://doi.org/10.1111/13652664.13487

Vidaller C., Dutoit T. \& Bischoff A. (sous presse). - Quels facteurs limitent l'installation d'une graminée pérenne dominante (Brachypodium retusum (Pers.) P. Beauv) pour la restauration écologique des pelouses sèches méditerranéennes?, in GOURDAIN P. (éd.), REVER 10 - 10ème Colloque du Réseau d'Échange et de Valorisation en Écologie de la Restauration, Paris, 19-21 mars 2019. Naturae 2021.

Vivier A., Rolan-Meynard M., Tuaux S., Rigault B., Reyjol Y., Melun G. \& Jourdan S. (sous presse). - Principes fondamentaux et exemple d'application du Guide pour l'élaboration des suivis d'opérations de restauration hydromorphologique en cours d'eau, in GOURDAIN P. (éd.), REVER 10 - 10ème Colloque du Réseau d'Échange et de Valorisation en Écologie de la Restauration, Paris, 19-21 mars 2019. Naturae 2022.

Soumis le 20 mai 2020; accepté le 2 novembre 2020 publié le 8 septembre 2021. 